Journal of Universal Computer Science, vol. 27, no. 8 (2021), 793-795

submitted: 23/7/2021, accepted: 25/7/2021, appeared: 28/8/2021 CC BY-ND 4.0

\title{
Recent Advances in Cybersecurity and Safety Architectures in Automotive, IT, and Connected Services \\ J.UCS Special Issue
}

\author{
Richard Messnarz \\ (ISCN GesmbH, Graz, Austria, \\ https://orcid.org/0000-0002-0555-3160,rmess@iscn.com) \\ Ricardo Colomo Palacios \\ (Ostfold University College, Halden, Norway, \\ https://orcid.org/0000-0002-1555-9726, ricardo.colomo-palacios@hiof.no) \\ Georg Macher \\ (TU Graz, Graz, Austria, \\ https://orcid.org/0000-0001-9215-3300,georg.macher@tugraz.at) \\ Andreas Riel \\ (Grenoble Institute of Technology, Grenoble, France, \\ https://orcid.org/0000-0001-9859-019X, andreas.riel@grenoble-inp.fr) \\ Miklos Biro \\ (Software Competence Center Hagenberg, Austria, \\ https://orcid.org/0000-0001-8627-1159, miklos.biro@scch.at)
}

\begin{abstract}
This is a special issue in cooperation with EuroSPI (www.eurospi.net). EuroSPI represents a large international network of renowned experts and annual European conference series running successfully since its foundation in 1994. From 2013 onwards, an international functional safety and from 2016 onwards a functional safety and cybersecurity workshop has been established, to which leading European and Asian industry and research have been actively contributing to.

High-quality, original papers about best practices for implementing functional safety and cybersecurity in automotive, IT, and connected services have been selected for this special issue. They provide insights into the current state of the art implementations in automotive safety and cybersecurity, IT security, and safety in future highly autonomous self-learning vehicles.
\end{abstract}

Keywords: Automotive Safety, Automotive Cybersecurity, IT Security, Cybersecurity, Autonomous Vehicles, Verification, Validation, Best Practice Design Patterns

Categories: D.2.1, D.2.2, D.2.5, D.4.6

DOI: $10.3897 /$ jucs.72027

\section{Introduction}

The server and software architectures in modern vehicles, IT systems [ISO27000 2018], and connected services are changing to fulfil the growing demands for cybersecurity 
and safety [ISO 214342020 ][ISO 26262 2011] [ISO 26262 2018]. More than $90 \%$ of functions in cars are controlled by software, and cybersecurity attacks targeting safety functions can lead to disasters. Up-to-date defensive software architectures need to deal with this. They requires the implementation of new software and hardware design patterns [AVSC 2020] [UL4600] [UNECE 2020] for highly automated software-based systems, as well as the development and training of new skills for the future design of complex systems of systems.

Every year, the EuroSPI conference series has contributions coming from at least 24 different countries, and moderates an annual workshop about functional safety and cybersecurity since 2016. In this special issue, $50 \%$ of the articles represent extended papers from that workshop community [Kreiner et al. 2016][Stolfa et al. 2017] [Larrucea et al. 2018] [Walker et.al. 2019] [Yilmaz 2020].

The SOQRATES group represents a working party of 24 large and medium-sized leading German and Austrian automotive and electronics companies, as well as and universities who cooperate in the elaboration of best practices for cybersecurity development and operation methods to cope with the forthcoming cybersecurity homologation processes for road vehicles and other mobility devices. From July 2022 onwards, vehicles cannot be released for road operation anymore without a successful cybersecurity assessment [UNECE 2020]. This influential group actively contributed to many of the articles in this special issue.

Leading industry like VOLKSWAGEN AG and SIEMENS AG responded to the J.UCS call and contributed key knowledge about how to make systems safe and secure.

\section{Overview of the Contributions to this Special Issue}

Masao Ito from Japan discusses the new UL 4600 standard and how it can be interpreted for describing self-driving scenarios for vehicle operations in a way these can be verified. Modern vehicles with a central gateway and artificial intelligence are programmed according these scenario specifications.

Svatopluk Stolfa, Jakub Stolfa, Petr Simonik, Tomas Mrovec and Tomas Harach describe the approach of a safety decomposition on a vehicle level, including the programming and adaptation of test vehicle scenarios, to make self-driving possible for medium-prized cars while still fulfilling the new UNECE regulations.

Jürgen Dobaj, Damjan Ekert, Jakub Stolfa, Svatopluk Stolfa, Richard Messnarz and Georg Macher discuss the threat and risk analysis and derived design patterns to achieve cybersecurity objectives based on a steering system example.

Damjan Ekert, Jürgen Dobaj and Alen Salamun discuss how cybersecurity verification and validation testing is performed in modern vehicles.

Alexander Poth, Mario Kottke, Kerstin Middelhauve, Torsten Mahr and Andreas Riel describe a framework to support adequate lean governance for implementing IT security standards in a large company.

Nermin Kajtazovic, Peter Hödl and Leo Happ Botler describe language and compiler strategies to be considered in mixed-criticality situations.

Hence, the selection of journal articles addresses different viewpoints on how to handle cybersecurity and safety, starting from the conceptual layer down to the detailed level, and covering both the embedded systems as well as the required IT infrastructure. 


\section{References}

[AVSC 2020] SAE ITC, AVSC00002202004: AVSC Best Practice for Describing an Operational Design Domain: Conceptual Framework and Lexicon, 2020.

[ISO 21434 2020] ISO 21434ISO/SAE 21434 DIS, Road vehicles - Cybersecurity engineering, DIS version, 2020

[ISO 26262 2011] ISO - International Organization for Standardization. "ISO 26262 Road vehicles Functional Safety Part 1-10”, 2011.

[ISO 26262 2018] ISO - International Organization for Standardization. "ISO CD 26262-2018 2nd Edition Road vehicles Functional Safety", 2018.

[ISO27000 2018] Information technology - Security techniques - Information security management systems - Overview and vocabulary - https://www.iso.org/standard/73906.html.

[Kreiner et al. 2016] Kreiner, C., Poth., A., O'Connor, R.V., and Messnarz R. (Eds), Systems, Software and Services Process Improvement, CCIS 633, Springer-Verlag, 2016.

[Stolfa et al. 2017] Stolfa, J, Stolfa, S., O'Connor, R.V., and Messnarz R. (Eds), Systems, Software and Services Process Improvement, CCIS 633, Springer-Verlag, 2017.

[Larrucea et al. 2018] Larrucea, X., Santamaria, I., O'Connor, R.V., Messnarz, R. (Eds), Systems, Software and Services Process Improvement, CCIS Vol. 896, Springer-Verlag, 2018.

[UL4600] Underwriters Laboratories (UL), UL 4600 Safety Standard for Autonomous Vehicles, 2020.

[UNECE 2020] UNECE, World Forum for Harmonization of Vehicle Regulations, Report of the Working Party on Automated/Autonomous and Connected Vehicles, Regulation ECE/TRANS/WP.29/GRVA/5, 2020.

[Walker et al. 2019] Walker A., O'Connor, R.V., Messnarz, R. (Eds), Systems, Software and Services Process Improvement, CCIS Vol. 1060, Springer-Verlag, 2019.

[Yilmaz 2020] Yilmaz M., Niemann J., Clarke P. Messnarz R., (Eds), Systems, Software and Services Process Improvement, CCIS Vol. 1251, Springer-Verlag, 2020. https://doi.org/10.1007/978-3-030-56441-4_34 